\title{
Partizipative Modellierung. Beteiligungsexperimente in der sozialökologischen Forschung
}

\author{
Alexander Bogner • Veronika Gaube $\cdot$ Barbara Smetschka
}

Zusammenfassung: Folgt man aktuellen soziologischen Diagnosen, so lässt sich heute in verschiedenen Gesellschaftsbereichen eine Aufwertung des Partizipationsgedankens konstatieren. Im Umweltbereich kommt es dabei, so unsere These, zu einer Experimentalisierung von Partizipation. Das heißt, neben traditionelle und zuweilen protestförmige Teilhabeforderungen ökologisch bewegter BürgerInnen treten neue Beteiligungsformate, die sich oft der Initiative seitens der Wissenschaft verdanken. Solche Beteiligungsexperimente werden in der sozialökologischen Forschung genutzt, um konkrete Lösungen im Bereich nachhaltiger Entwicklung zu konzipieren und umsetzbar zu machen. Unsere empirische Analyse zeigt, dass die mit Partizipation erwarteten Rationalitätsgewinne am Ehesten in solchen Kontexten zu erwarten sind, in denen Eigeninteresse, lebensweltliche Betroffenheit und ein Spezialwissen der Beteiligten vorausgesetzt werden können.

Schlüsselwörter: BürgerInnenbeteiligung · Modellierung · Sozial-Ökologische Forschung · Protest $\cdot$ Transdisziplinarität

\section{Participatory Modelling. Experiments in Participation for Socio-Ecological Research}

Abstract: Results of contemporary sociological studies suggest an on-going revaluation of the idea of participation in different areas of society. We argue that in environmental issues public participation increasingly becomes experimental. This means that actors mainly from science initiate and organise participation. These new forms of participation evolve in addition to traditional calls for participation which sometimes take on the form of protest by ecologically moved citizens. For

\author{
Alexander Bogner $(\square)$ \\ Strohgasse 45 \\ 1030 Wien; ÖSTERREICH \\ E-Mail: abogner@oeaw.ac.at \\ Barbara Smetschka $(\bowtie)$ \\ Alpen-Adria Universität Klagenfurt \\ Institut für Soziale Ökologie \\ Schottenfeldgasse 29/5 \\ 1070 Wien, ÖSTERREICH \\ E-Mail: barbara.smetschka@aau.at
}

Veronika Gaube $(\triangle)$

Österreichische Akademie der Wissenschaften Alpen-Adria Universität Klagenfurt

Institut für Technikfolgen-Abschätzung Institut für Soziale Ökologie

Schottenfeldgasse 29/5

1070 Wien, ÖSTERREICH

E-Mail: veronika.gaube@aau.at 
socio-ecological research, experiments in participation are useful to develop concrete and applicable solutions in the field of sustainable development. Based on empirical research we show that the hoped-for gains in rationality attached to lay people participation materialises if the citizens' self-interest is touched, if problems immediately afflict their life-world, and if participants can contribute special knowledge.

Keywords: Public Participation · Modelling · Socio-Ecological Research · Protest · Transdisciplinarity

Partizipation hat Hochkonjunktur. Folgt man aktuellen soziologischen Diagnosen, so lässt sich heute in ganz verschiedenen Bereichen der Gesellschaft eine Aufwertung des Partizipationsgedankens konstatieren.

Die Industrie- und Arbeitssoziologie etwa beobachtet, dass im Betrieb nicht länger einseitig hierarchisch vorgegeben oder technisch-ökonomisch berechnet werden kann, was als Leistung gilt. Dies muss vielmehr unter Einbezug der Beschäftigten ausgehandelt werden. Auf diese Weise treten in der betrieblichen Leistungspolitik partizipative Begründungsmuster neben etablierte Legitimationsformen, wie z. B. tayloristische oder marktbezogene (Menz 2009). Die Gesundheits- und Medizinsoziologie stellt fest, dass es im Zuge der „Genetifizierung“ der Medizin zu einer Überforderung der handlungsleitenden Dichotomie von gesund und krank kommt. Aus den damit verbundenen Unsicherheiten resultiert ein subtiler Zwang zur Einbeziehung der KlientInnen: Man setzt zunehmend auf Dialog und Deliberation, um den Einsatz medizinischer Diagnostik und Therapie zu dosieren (Bogner 2005).

Die Techniksoziologie registriert in ähnlicher Weise einen anhaltenden Trend zur Bürgerbeteiligung. Im Zuge diverser Technikkontroversen hat sich ein Kanon „demokratischer Technikbewertung“ etabliert (Abels und Bora 2004), der den Versuch zum Ausdruck bringt, in Form institutionalisierter oder informeller Beteiligungsverfahren auch Laien eine Stimme zu geben. In der Stadtplanung beobachtet man seit den 1990er Jahren die Integration direkt-demokratischer Partizipationsinstrumente in Planungsprozesse. Mittlerweile hat sich ein kooperatives Planungsverständnis weitgehend durchgesetzt (Dröge und Magnin 2010). Fast scheint es, als seien wir auf dem Weg in die Partizipationsgesellschaft, und zwar in dem Sinne, dass Bürger in vormals partizipationsverschlossene, expertendominierte Bereiche eindringen - oder auch: zur Partizipation eingeladen werden.

Welche Tendenzen lassen sich nun im Umweltbereich beobachten, also in einem Bereich, der geradezu paradigmatisch für Beteiligungsforderungen und -prozesse ist? Unsere zentrale These lautet, dass es in Umweltfragen zu einer Experimentalisierung von Partizipation kommt. Das heißt, es lässt sich heute eine Ausdifferenzierung von Beteiligungsformen und -zwecken beobachten. Auf der einen Seite können sich die Teilhabeund Gestaltungsansprüche der BürgerInnen als Protest artikulieren, so wie dies für die Hochzeiten der Ökologiebewegung typisch war (Kap. 1). Auf der anderen Seite führen verschiedenartige Versuche, die Rationalität der Laien für die Politikberatung und, vor allem, für die wissenschaftliche Forschung produktiv zu machen, zu neuen Beteiligungsformaten. Dies kann man analytisch als Experimentalisierung von Partizipation 
begreifen (Kap. 2). Solche Beteiligungsexperimente werden in der sozialökologischen Forschung genutzt, um konkrete Lösungen im Bereich nachhaltiger Entwicklung zu konzipieren und umsetzbar zu machen (Kap. 3). Dabei zeigt unsere empirische Analyse, dass die mit Partizipation erwarteten Rationalitätsgewinne am ehesten in solchen Kontexten zu erwarten sind, in denen Eigeninteresse, lebensweltliche Betroffenheit und ein Spezialwissen der Beteiligten vorausgesetzt werden kann (Kap. 4).

\section{Partizipation als Protest}

Umweltfragen und Umweltkonflikte sind seit jeher ein besonders attraktiver Bereich für soziologische Analysen von Beteiligungsprozessen. Dies ist nicht zuletzt darin begründet, dass hier politische Teilhabeforderungen „von unten“ artikuliert und organisiert wurden (z. B. in Form von Bürgerinitiativen) und dass diese Beteiligungsansprüche sich oftmals als Protest konstituierten, besonders eindrucksvoll im Streit um die Kernenergie (Roose 2010). In den Hochphasen des Streits um die Kernenergie entwickelten sich Massendemonstrationen, die - wie in Brokdorf oder Wackersdorf - über hunderttausend TeilnehmerInnen anzogen. Auch wenn dieser Protest meist friedlich verlief, so zeichnete sich gerade die frühe Anti-Atomkraftbewegung durch einen hohen Grad an Militanz aus (Rucht 2003, S. 163).

Partizipation als Protest heißt demnach: Selbstkonstituierung themenspezifischer Teilöffentlichkeiten zur Artikulation und Durchsetzung politischer Interessen vor dem Hintergrund (bzw. mit der Folge) einer entsprechenden Politisierung von Umweltfragen bzw. der „Natur“. ${ }^{1}$ Diese Protestpartizipation - ob militant oder friedlich - ist auf reale Konflikte und klare politische Teilhabebestrebungen bezogen. Angesichts von Massendemonstrationen, Bürgerinitiativen und Volksbegehren bedarf es entweder gar keiner Einladung zur Partizipation; und da, wo sie auf Einladung stattfindet (z. B. Schlichtungs- oder Dialogverfahren), realisiert sie sich vor dem Hintergrund einer breiteren Mobilisierung und realer Kontroversen. ${ }^{2}$ In diesem Sinn kann man die Auseinandersetzungen um Kernenergie, Kraftwerke (wie Hainburg) oder Kopfbahnhöfe (Stuttgart 21) als Varianten einer Protestpartizipation verstehen. Protest war ein wichtiger Katalysator für die Herausbildung einer Umweltbewegung, die sich ab den frühen 1970er Jahren zu etablieren begann, wobei sich diese Protestbewegung als wissenschaftlich informierte Ökologiebewegung verstand - im Gegensatz zur lebensphilosophisch geprägten Naturschutzbewegung der Jahrhundertwende (mit Blick auf Österreich: Schmid und Veichtlbauer 2007).

Die Ökologiebewegung war nicht zuletzt für die soziologische Theoriebildung von großer Bedeutung. Ulrich Becks Diagnose einer „Risikogesellschaft“ (1986) ist ohne die breitenwirksame Thematisierung nicht-intendierter Modernisierungsfolgen seitens der Ökologiebewegung nicht zu denken. Und Niklas Luhmann hat die Resonanz des ökologischen Protests zum Anlass für die Frage genommen, ob sich vermittels des Protests nicht Kommunikationssysteme eigener Art stabilisierten: Protestbewegungen. ${ }^{3}$ Auf seine Theorie des Protests wollen wir im Folgenden etwas genauer eingehen, weil hier Funktion und Folgen von Protest in gesellschaftstheoretisch-systematischer Art bestimmt werden. Seine Konzeption des Protests als einer Form der Differenzerhaltung er- 
laubt es in Hinblick auf die von uns angestrebte Unterscheidung von Beteiligungsformen in Umweltfragen, eine wichtige Unterscheidung einzuziehen: jene zwischen kommunikativen Normal- und Sonderformen von Beteiligung.

Proteste bestimmt Luhmann ganz allgemein als Kommunikationen, „die an andere adressiert sind und deren Verantwortung anmahnen.“ (Luhmann 1991, S. 135) Protest setzt damit immer eine andere Seite voraus, die zu reagieren hat. Diese Unterscheidung zwischen einem „Wir“ und „den anderen“" markiert eine spezifische Art und Weise der Differenzziehung. Ob diese Form der Differenzziehung, also der Protest, allerdings systembildend wirkt, ist offen. Im Fall der Ökologiebewegung - als ein Beispiel erfolgreicher Systembildung - stellt die Form des Protests gewissermaßen das funktionale Äquivalent für den Code der Funktionssysteme dar; die (fluktuierenden und daher im Prinzip auswechselbaren) Themen der Ökobewegung entsprechen in Luhmanns Systemlogik Programmen, die festlegen, gegen wen protestiert wird. In Funktionssystemen repräsentieren solche Programme Prozessierungsregeln, die festlegen, ob die eine oder die andere Seite der Unterscheidung aktualisiert wird. Im Fall der Wissenschaft sind das Theorien, im Fall des Rechts Rechtsverordnungen. Diese Parallelisierung kommt jedoch im Fall funktionssystemspezifischer Codes an ihre Grenzen. Denn Codes sind inhaltlich festgelegt (z. B. wahr/falsch) und beziehen daraus ihren Reflexionswert. Die Wissenschaft fragt entsprechend: Ist diese oder jene Behauptung wahr oder unwahr? Der dem Protest zugrunde liegende Schematismus ist daher nicht im strengen Sinne als Code zu verstehen, weil der Negativwert nicht zur Reflexion des Positivwerts - nämlich des ökologischen Wissens der Protestierenden - dient (Luhmann 1996, S. 177). Mit anderen Worten: Im Fall des Protests unterbleibt die Reflexion auf die womöglich guten Gründe der Gegenseite - Luhmanns Hauptvorwurf an die Adresse der Ökologiebewegung. ${ }^{4}$ Aus differenzierungstheoretischer Perspektive macht Protest also eine Unterscheidung, die blind macht für die eigenen Blindflecken: Man konstruiert eine privilegierte Beobachtungsposition, die mit der Emphase der Ganzheitlichkeit zwar verspricht, das Problem in all seiner Komplexität erkennen zu können, tatsächlich jedoch die sprichwörtliche Unübersichtlichkeit der Moderne unterschätzt. Ein solcher Holismus (und bekannte Surrogate wie die „freischwebende Intelligenz“") wird durch gesellschaftliche Differenzierung freilich unplausibel: „Das Ganze kann nicht zugleich Teil des Ganzen sein.“(Luhmann 1986, S. 227)

Aus differenzierungstheoretischer Perspektive wird man von einem allmählichen Versickern des Protests ausgehen. Und im Prinzip dürfte wohl gelten, dass sich die ökologiebewegte Protestbewegung weitgehend institutionalisiert und thematisch ausdifferenziert hat (vgl. Take 2002). „Die Umweltbewegung“, so resümiert Karl-Werner Brand (2008, S. 242), ,hat sich (. . .) mehr und mehr von einer Protest- zu einer proaktiven Umsetzungsbewegung gewandelt. Spektakuläre Aktionen und große Demonstrationen sind einem professionellen, lösungsorientierten Engagement auf verschiedensten Ebenen gewichen." Dennoch: Der erneute und durch die japanische Nuklearkatastrophe intensivierte Streit um die Kernenergie zeigt, dass die längere Zeit „,im Halbschlaf befindliche Bewegung“ (Rucht 2008, S. 266) durchaus reaktivierungsfähig ist. Und auch der Protest gegen den Umbau des Stuttgarter Hauptbahnhofs zu einem Durchgangsbahnhof macht deutlich, dass Umweltthemen - in diesem Fall: der Schutz alter Bäume und die Qualität der Mineralwasserquellen - nach wie vor Mobilisierungskraft haben. Ein drittes Bei- 
spiel für die Nachhaltigkeit des ökologischen Protests wäre die regelmäßige Mobilisierung im Wendland gegen die Castor-Transporte.

Freilich: Im Unterschied zu den 1980er Jahren sind die Proteste heute weniger radikal (und zwar sowohl in ihren Forderungen als auch in ihren Aktionsformen); sie bleiben meist lokal begrenzt, und vor allem: Sie müssen im Regelfall ohne kohärente Ideologie und vergemeinschaftende Vision auskommen. Diese Diversität der Umweltbewegung wird in Deutschland derzeit vor allem in Energiefragen anschaulich: Es gibt Demonstrationen und Plakataktionen für und gegen Kernenergie, Proteste für und gegen Erdkabel, Bürgerinitiativen für und gegen Wind- und Solarparks - und alles jeweils aus ökologischen Gründen.

Diese Diversifizierung von Themen und Protestgründen lässt auf gesellschaftliche Differenzierung schließen. Zwar gibt es offensichtlich zunehmend mehr Gelegenheiten für ökologischen Protest, doch wird dieser zunehmend heterogen, unübersichtlich und tendenziell kleinschrittig, weil lösungsorientiert. Auf diese Weise drohen die Themen nicht länger attraktiv zu wirken. Der Impetus (oder die Illusion) der „Systemveränderung" geht verloren. Man reformiert an Details herum und trifft dabei auf die ebenfalls reformwilligen „Gegner“ von gestern. Darüber hinaus lässt sich eine zweite Ursache für die Absorption von Protest denken: der Erfolg der Protestbewegungen. Öko-Audits für Organisationen, Umweltbeauftragte in Ämtern und Behörden, mindestens eine grüne Partei pro Land: All dies macht deutlich, dass der Erfolg - in Form der „Ökologisierung“ von Wirtschaft, Politik und Medien - ein ähnlich ernstes Problem für die Ökologiebewegung darstellt wie die Erfolglosigkeit.

All diesen Erwägungen zum Trotz lässt sich - gerade gegenwärtig - eine gewisse Attraktivität ökologischen Protests nicht leugnen. Doch ob die Proteste nun zu- oder abnehmen, ist im Hinblick auf die von uns angestrebte Unterscheidung von Partizipationsformen im Umweltbereich nicht weiter relevant. Es geht vielmehr darum, den Protest als eine spezifische Kommunikationsform verständlich zu machen, um eigenen Interessen und Anliegen Gehör zu verschaffen. Solche Beteiligungsansprüche artikulieren sich heute nicht mehr allein auf dem kommunikativen Sonderweg des Protests, also gewissermaßen von außen. Wir beobachten vielmehr den Einbezug von Laien (als BürgerInnen, Betroffene oder KonsumentInnen) in den Kernbereichen ökologisch motivierter Forschung, Technologieentwicklung und Politik. Dieses Einschwenken von Partizipation auf die kommunikativen Normalwege ist Gegenstand des nächsten Abschnitts.

\section{Partizipation als Experiment}

Die eingangs formulierte These, dass es in Umweltfragen zu einer Experimentalisierung von Partizipation kommt, bezieht sich auf die Beobachtung einer Ausdifferenzierung von Beteiligungsformen und -zwecken. Über protestförmige Partizipation haben wir gesprochen. Was lässt sich nun unter experimenteller Partizipation verstehen? Eine Experimentalisierung von Partizipation lässt sich im Rückgriff auf zwei soziologische Beiträge aus jüngster Zeit argumentieren: zum einen mit Bezug auf das Konzept der „Realexperimente“ (Krohn 2007), zum anderen mit Rekurs auf das Stichwort einer „Partizipation als Laborexperiment" (Bogner 2010). 


\subsection{Laborpartizipation}

Im Protest, so haben wir gesehen, artikuliert sich ein relativ klar definiertes Partizipationsinteresse „,von unten“. Ein solches Interesse lässt sich jedoch nicht immer (und heute vielleicht sogar: immer weniger) voraussetzen. Wir erleben darum auch und gerade im Umweltbereich verstärkt Formen einer Partizipation, die sich als „Laborexperiment“ verstehen lässt (Bogner 2010). Partizipation als Laborexperiment heißt: eine von „,von oben", also durch Partizipationsprofis organisierte und unter aufwändiger methodischer Kontrolle statt findende Beteiligung, die weitgehend ohne Bezug auf öffentliche Kontroversen, politische Teilhabebestrebungen und individuelle Betroffenheiten abläuft sowie fernab konkreter Handlungszwänge und realer Gestaltungsprozesse - also gewissermaßen in einem von der Gesellschaft abgeschirmten Raum (vgl. zur Dekontextualität des Laborexperiments und soziologischen Gegenentwürfen einer „kontextualisierten“ Wissenschaft Bon $\beta$ et al. 1993). Es sind private PartizipationsunternehmerInnen, nicht selten aus dem Bereich der Sozialwissenschaften, die Prozesse der Bürgerbeteiligung initiieren, organisieren und inszenieren. Oftmals realisiert sich diese Beteiligung in Form von drittmittelfinanzierten Forschungsprojekten, und zuweilen ist gar nicht mehr auszumachen, ob das primäre Interesse der Mobilisierung von Laien oder der sozialwissenschaftlichen Beobachtung dieser Experimente gilt. Die methodische Kontrolle sowie ihre Herausgehobenheit aus gesellschaftlichen Zusammenhängen, also ihre Dekontextualität oder „Künstlichkeit“, sind zentrale Merkmale dieser Verfahren.

Eine solche Laborpartizipation lässt sich empirisch derzeit vor allem an jenen Beteiligungsexperimenten beobachten, die mit dem Ziel der Politikberatung in großen Umweltfragen initiiert werden. An erster Stelle stehen hier moderierte Deliberationsverfahren wie die medienorientierte Bürgerkonferenz, die nicht dazu dienen, ein existierendes Protestpotenzial zu kanalisieren; vielmehr geht es darum, die Öffentlichkeit überhaupt erst einmal für das betreffende Thema zu interessieren. ${ }^{5}$

Diese Entwicklung ist nicht zuletzt vor dem Hintergrund einer Vorverlagerung der Partizipation in sachlicher und zeitlicher Hinsicht zu verstehen. Das entsprechende Stichwort lautet „upstream engagement“" (Wilsdon und Willis 2004). Dahinter steckt der Versuch, Beteiligungsprozesse in einem frühen Stadium von Planungs- und Innovationsprozessen zu initiieren, um die BürgerInnen nicht mit fertigen Produkten, finalisierten Forschungsprogrammen oder irreversiblen Infrastrukturprojekten zu konfrontieren. Diese Bemühungen um Einbeziehung zu einem frühen Zeitpunkt werden derzeit verstärkt im Forschungsbereich, insbesondere in Hoffnungsgebieten wie der Nanotechnologie sichtbar (Gavelin et al. 2007). Derartige Präventionsstrategien finden sich im Rahmen einer ,,anticipatory governance“, aber auch im Bereich der Klimaanpassung (Dessai und Hulme 2004). Hinter diesen Bemühungen steht die Annahme, dass viele Umweltund Technikkonflikte der Vergangenheit sich nicht zuletzt aus dem Protest gegen intransparente und expertokratische Entscheidungsprozesse gespeist haben. Allerdings verdankt sich diese Laienmobilisierung, wie aktuelle Beispiele zeigen, ausschließlich der Initiative ,von oben“ (Kurath und Gisler 2009).

Vergleichbare Partizipationsinitiativen sind auch im Umweltbereich zu beobachten und auch dort gerade in Fällen, wo die erhoffte Partizipation ausbleibt. Schon in der 1992 verabschiedeten Klimarahmenkonvention der Vereinten Nationen wird auf die Sti- 
mulierung von Partizipationsprozessen seitens der Regierungen gesetzt, um adäquate Klimastrategien zu entwickeln (UNFCCC 1992). Im Kyoto-Protokoll werden die Vertragsstaaten darauf verpflichtet, Maßnahmen zu ergreifen, um das öffentliche Bewusstsein und die Teilhabe der BürgerInnen am Klimadiskurs zu ermöglichen. „Public participation in decision-making", resümieren denn auch Few et al. (2007, S. 48), ,is now a commonly stated objective across most sectors of environmental policy." Gleichzeitig sind die BürgerInnen eigenartig inaktiv, wenn es um die großen und komplexen Umweltfragen der Gegenwart wie z. B. den Klimaschutz geht (Walk 2010). Es existieren zwar gut organisierte Interessenvertretungen (NGOs). Doch es gibt derzeit - gerade in den medienwirksamen Fragen von Klimawandel, Biodiversität und Energie - keine neue, politisch wirksame Protestbewegung, die reale Teilhabe- und Gestaltungsforderungen ,von unten“ zum Ausdruck bringen würde.

Stattdessen werden Beteiligungs- und Deliberationsverfahren von Seiten interessierter WissenschaftlerInnen - oft aus dem Nachhaltigkeitsbereich oder der Technikfolgenabschätzung - organisiert, um einen BürgerInnendiskurs über Umweltfragen überhaupt erst einmal anzustoßen. Diese Experimente nehmen teilweise gigantische Ausmaße an. So wurde im Herbst 2009 ein globales Partizipationsexperiment zum Thema Klimawandel durchgeführt. Im Vorfeld des UN-Gipfels zum Klimawandel, der im Dezember 2009 in Kopenhagen stattfand, diskutierten in weltweit 44 Bürgerkonferenzen insgesamt rund 4400 Laien aus 38 Ländern über die Folgen des Klimawandels und wirksame Politikstrategien. Dieses Beteiligungsverfahren wurde vom Dänischen Technologierat mithilfe von über 50 Partnerinstitutionen in Form eines Projekts aufgesetzt (www.wwviews.org). Ziel dieses Projekts war es, den vom Klimawandel betroffenen, aber bislang nicht diskursrelevanten BürgerInnen eine Stimme zu geben. In Österreich waren 96 BürgerInnen beteiligt. Im Rahmen einer eintägigen Veranstaltung entwickelten sie ihre Empfehlungen. Die in dem weltweit synchronisierten Prozess entstandenen „Policy Recommendations“ wurden auf der Klimakonferenz in Kopenhagen präsentiert. Eine nennenswerte Resonanz erzielten die (recht konventionellen) Empfehlungen dieser Weltbürgerschaft nicht, weder auf internationaler noch auf nationaler Ebene (Bechtold et al. 2011).

Zwei weitere Bürgerkonferenzen wurden zu umweltpolitischen Themen bislang in Österreich durchgeführt: 1997 zum Thema „Bodennahes Ozon“, 2009 zum Thema „Risiko Energiegesellschaft". Letztere fand im Rahmen der vom Umweltbundesamt und Radio Ö 1 getragenen Initiative „Risiko:dialog“" statt (www.risikodialog.at). 32 BürgerInnen, die nach Diversitätsgesichtspunkten ausgewählt worden waren, erarbeiteten ein „Energie-Kommuniquéc, das 45 Empfehlungen an die Politik enthält. Diese Empfehlungen tragen in ihrer inhaltlichen Spezifität (z. B. Energie-Contracting für Private, nach Verbrauch gestaffelte Stromtarife, Produktkennzeichnung nach Energieeffizienzklassen) deutlich die Handschrift der ExpertInnen. Im Laufe des Jahres 2009 wurde das Bürgervotum den Vertretern einiger Landesregierungen, verschiedener Bundesministerien und zahlreicher Interessenvertretungen (u. a. Österreichischer Gewerkschaftsbund, Industriellenvereinigung, NGOs) übergeben. Wie in vielen anderen Fällen auch, lässt sich ein geringes Interesse an dieser weder protestförmigen noch institutionalisierten Beteiligungsform feststellen. 


\subsection{Realexperimente}

Das Konzept der Realexperimente basiert auf der Beobachtung, dass vormals institutionell eingehegte Praktiken der Wissensproduktion auf die gesamte Gesellschaft ausgreifen. Weil Umwelteingriffe oder Technikfolgen meist nicht im Labormaßstab studiert werden können, komme es in zunehmendem Maße zu Experimenten innerhalb und mit der Gesellschaft (Krohn und Weyer 1989). In der aktuellen Diskussion um Realexperimente geht es dabei vor allem um Gestaltungsexperimente im Bereich der ökologischen Sanierung und Renaturierung (Groß et al. 2003). Im Kern handelt es hier um Natureingriffe mit gezielten Gestaltungsabsichten und systematischen - durch das Projektdesign garantierten - Lernchancen. Ökologische Fragen markieren denn auch jenen Bereich, wo verstärkt Partizipation ins Spiel kommt. So lassen sich ökologische Realexperimente mit Krohn (2007) als eine neuartige und sozial robuste Form der Wissensproduktion verstehen, die im Kontext der Wissensanwendung mit einem bestimmten Gestaltungsziel und unter Beteiligung heterogener AkteurInnen - oft von Laien - abläuft. Das Erfahrungswissen der Laien vor Ort erhält demnach im Prinzip eine ähnlich zentrale Bedeutung wie das theoretische Wissen der ForscherInnen. Das heißt, anders als Laborexperimente sind Realexperimente durch ihre Einbettung in konkrete Gestaltungsprozesse und die Ermöglichung von Lernchancen durch das Unterlaufen etablierter Grenzziehungen (Laie/Experte, Wissensproduktion/Wissensanwendung) gekennzeichnet.

Dass man Realexperimente zunehmend als Normalform gesellschaftlichen Lernens verstehen müsse, wie Groß et al. (2005) prognostizieren, lässt sich anhand einschlägiger Beispiele aus dem Umweltbereich zeigen. Eine weithin beachtete und beforschte Praxis ist die NutzerInnenbeteiligung bei „,Nachhaltigkeitstechnologien “. Mit der partizipativen Gestaltung und Entwicklung umweltsensitiver Technologien verbindet sich die Annahme, dass BürgerInnenbeteiligung in Innovationsprozessen die Akzeptanz von Produkten erhöht und darüber hinaus zur Verbesserung der neuen Technologien beiträgt. Diese Idee wurde im niederländischen Ansatz des „Constructive Technology Assessment" konzeptionell ausformuliert (Schot und Rip 1996). Seitens der Forschung, Planungsbehörden oder Firmen wird die spezialisierte Laienexpertise als Ideenpool genutzt, um, im Sinne von Nowotny et al. (2001), sozial robustes Wissen zu produzieren zunächst auf lokaler Ebene, aber mit dem Ziel der Generalisierbarkeit. Diese Beteiligungsvariante umfasst ein weites und heterogenes Anwendungsgebiet. Dementsprechend variiert auch die Innovationsfunktion der beteiligten AkteurInnen: Das Spektrum reicht von eigenständigen Innovationen durch Laienexpertise (z. B. bei Windkraftanlagen: Jørgensen und Karnøe 1995) über inkrementelle Verbesserungen (z. B. bei Biomasseheizungen: Rohracher 2002) bis hin zu gemeinschaftlichen Planungsprozessen auf Basis etablierter Technologien (z. B. ökologische Wohnkonzepte: Ornetzeder und Rohracher 2006). Dabei treten die NutzerInnen in unterschiedlichen Rollen in Erscheinung: als ErfinderInnen, PlanerInnen und OrganisatorInnen. In jedem Fall beziehen sich derartige Beteiligungsexperimente auf Situationen und Kontexte, in denen ein Interesse und Engagement der BürgerInnen erwartet werden kann - sei es, weil sie, wie beim ökologischen Bauen, als unmittelbar Betroffene adressiert werden, sei es, weil sie, wie bei der Windkraft- oder Solartechnologie, als selbst organisierte Interessengruppe in Erscheinung treten. 
Eine weitere interessante Variante der Realexperimente stellt die partizipative Modell- und Theorieentwicklung dar, also Laienbeteiligung im Kernbereich der humanund sozialökologischen Forschung. Diese Partizipationsvariante hat in den letzten Jahren erheblich an Bedeutung gewonnen, und es ist kein Wunder, dass die Nachhaltigkeitsforschung für manche fast schon zum Synonym für Transdisziplinarität geworden ist (Hadorn et al. 2006). Transdisziplinarität wird dabei im Sinne von Gibbons et al. (1994) als Forschung unter Beteiligung außerwissenschaftlicher AkteurInnen verstanden („Modus 2“ der Wissensproduktion). ${ }^{6}$ Mit Blick auf die vielfältigen Transdisziplinaritätsinitiativen im Bereich der Nachhaltigkeitsforschung (Pohl et al. 2010, S. 132) lässt sich denn auch ein Normalisierungseffekt konstatieren: Vom politischen Kampfbegriff im Dienste einer tief greifenden Reform von Wissenschaft, Universität und Gesellschaft (Jantsch 1972) ist Transdisziplinarität zu einer Beschreibungsformel für projektförmige, pragmatische und partizipative Forschung geworden, die mittlerweile zum Forschungsalltag gehört (vgl. Bogner et al. 2010). Weil sich derartige Projekte meist den Vorgaben der Forschungsförderung verdanken, resultiert die Laienbeteiligung im Regelfall aus der Initiative „von oben“ - allerdings auf Basis eines bereits artikulierten oder voraussetzbaren Beteiligungsinteresses und einer begründeten Aussicht auf einen spezifischen Mehrwert dieser Partizipation (z. B. empirisches Wissen).

\subsection{Rationalitätsgewinne}

Ob auf Politikberatung zielende Laborpartizipation oder im Bereich von Forschung und Technikentwicklung angesiedelte Realexperimente: Beiden Experimentalformen gemeinsam ist die Hoffnung auf Rationalitätsgewinne. Was heißt das? Die Diskussion um Rationalitätsgewinne durch Laienbeteiligung ist recht ausführlich in der Wissenschaftsforschung geführt worden. Empirische Bezugspunkte waren die produktive Rolle von PatientInnenorganisationen und AktivistInnen in der klinischen Forschung (Epstein 1996) sowie das kritische Potenzial von Laienexpertise in Umweltkonflikten (Wynne 1996). Stellvertretend für viele formuliert Sheila Jasanoff (2003, S. 397 f.): „Public engagement is needed in order to test and contest the framing of the issues that experts are to resolve. Without such critical supervision, experts have often found themselves offering irrelevant advice on wrong or misguided questions.“

Die Forderung nach Beteiligung verbindet sich demnach mit der systematisch begründeten Erwartung auf die Artikulation ,alternativer“ Rationalitäten. Dieser Begriff ist als Dachkategorie zu verstehen, die ihre spezifischen Konturen erst in der Entgegensetzung zu einer methodisch kontrollierten und daher systematisch Blindflecke erzeugenden Expertenvernunft gewinnt. Im Einzelnen fallen unterschiedliche Formen nicht wissenschaftlich gesicherten Wissens (praktisches oder lokales Wissen) darunter, aber auch lebensweltliche Erfahrungen oder die Formulierung neuer Problematisierungsperspektiven („Frames“). Für die (imaginierten) AdressatInnen aus Politik und Forschung ergibt sich daraus das Versprechen einer umfassenderen Expertise, einer Meinungsbildung auf breiterer Basis und damit letztlich die Chance zur Entwicklung ,robuster“ Entscheidungen.

Rationalitätsgewinne beziehen sich demnach - in Luhmann'schen Begriffen - auf 
Leistungen in der Sachdimension (also in der Verhandlung des Themas), nicht jedoch in der Sozialdimension (z. B. Geselligkeit, Vertrauensbildung). Dass beide Ebenen nur analytisch zu trennen sind, versteht sich, ist aber zu Untersuchungszwecken zielführend. So wird an unserem Forschungsbeispiel deutlich, dass Leistungen in der Sozialdimension (im Sinne von Vertrauensgewinnen) eine wesentliche Voraussetzung für das Gelingen des transdisziplinären Prozesses darstellen. Allerdings: Leistungen in der Sozialdimension werden den Laien ohnehin eher zugetraut als solche in der Sachdimension. Worin freilich die Leistungen in der Sachdimension im Einzelnen bestehen, hängt nicht zuletzt von der Funktion ab, die man dem Wissen und den Erfahrungen der Laien zuschreibt. So kann man Tatsachenbehauptungen der Laien gegebenenfalls als Kritik des Expertenwissens verstehen oder schlicht als Faktenwissen; die von den Laien aufgeworfenen Fragen und Perspektiven können den dominanten Bezugsrahmen, in dem ein Thema verhandelt wird, bestätigen oder in Frage stellen. In Summe ergeben sich Rationalitätsgewinne durch Partizipation, um es kurz zu bündeln, in der Herausforderung und Kritik oder aber - nach der Lesart von Collins und Evans (2007) - in der Ergänzung (,contributory expertise“) einer durch Professionalisierung verengten Expertenperspektive.

\section{Partizipative Modellentwicklung in der sozialökologischen Forschung}

Transdisziplinäre Forschung stellt ein Realexperiment dar, sofern darunter ein partizipatives Forschungsdesign unter Einschluss betroffener AkteurInnen verstanden wird, das den Aushandlungscharakter des Forschungsprozesses betont und durch Rückkopplungen (z. B. in Form von Feedback-Schleifen, Workshops u. ä.) systematische Lernchancen vorsieht (vgl. Newig et al. 2008; Reed et al. 2006). Einen solchen transdisziplinären Ansatz wollen wir im Folgenden anhand eines Beispiels darstellen, um daran die nahe liegende Frage nach dem Sinn und Mehrwert experimenteller Partizipation diskutieren zu können. Wir beziehen uns auf ein Projekt, das am Institut für Soziale Ökologie in Wien angesiedelt war und im Rahmen von „TRAFO - Transdisziplinäres Forschen“ des BMWF gefördert wurde. ${ }^{7}$ Im Zentrum dieses Projekts stand die Frage, ob Reformen im Agrarsubventionswesen - wie beispielsweise jene der Gemeinsamen Agrarpolitik (GAP) - geschlechtsspezifische Auswirkungen auf landwirtschaftliche Betriebe haben und infolgedessen Produktionsentscheidungen, die für die Landnutzung relevant werden, beeinflussen (Smetschka et al. 2008). Um die gesellschaftliche Relevanz und den sozialökologischen Aspekt dieser Fragestellung aufzuschlüsseln, bedarf es eines kurzen Exkurses zum Strukturwandel in der Landwirtschaft.

Die Landwirtschaft und damit der Zusammenhang zwischen Landnutzung und sozialem Wandel, ist eines der klassischen sozialökologischen Forschungsthemen. Der Einfluss biophysischer sowie sozialer und ökonomischer Faktoren (z. B. Familien- und Einkommensstrukturen usw.) auf die landwirtschaftliche Praxis ist dabei Forschungsgegenstand auf unterschiedlichsten Skalenebenen (global, national, regional). In Österreich sind heute weniger als sechs Prozent der Bevölkerung in der Landwirtschaft tätig. Landwirtschaftliche Aktivitäten bestimmen allerdings maßgeblich das Aussehen der österreichischen Landschaft, weil fast 80 Prozent der Gesamtfläche nach wie vor unter landwirt- 
schaftlicher Nutzung steht (BMLFUW 2010). Die Industrialisierung und die damit einhergehende Intensivierung der landwirtschaftlichen Produktion in den letzten 60 Jahren haben zu einem großräumigen Umstrukturierungs- und Konzentrationsprozess in der Landwirtschaft und Landnutzung beigetragen (Krausmann et al. 2003; Fischer-Kowalski und Haberl 2007). Die zunehmende Marktintegration der Landwirtschaft und die enorme Steigerung der Produktivität bewirkten zunächst eine Entlastung der landwirtschaftlichen Betriebe, da mit geringerem Mittel- und Arbeitszeiteinsatz mehr produziert werden konnte. Während die in den Gunstlagen angesiedelten Acker- und Veredelungsbetriebe durch die neuen Intensivierungsmöglichkeiten begünstigt werden und mit geringerem Arbeitseinsatz ökonomische Gewinne erzielen können, geraten allerdings jene Produktionsschwerpunkte in Ungunstlagen, von denen es aufgrund des alpinen Charakters weiter Teile Österreichs viele gibt, zunehmend unter Druck (Haberl et al. 2003; Krausmann et al. 2003). Beide Prozesse haben ökologische Folgewirkungen: Nimmt in den Gunstlagen die Verwendung von künstlichem Dünger zu, was Umweltprobleme wie Grundwasserverschmutzung nach sich zieht, so weiten sich in den Ungunstlagen die Waldflächen dramatisch aus, was ökologisch kein Problem ist, sehr wohl aber zu einem sozialen Problem werden kann, wenn die Lebensqualität in diesen Regionen sinkt (Gaube et al. 2009). Dementsprechend sind agrarische Subventionen zur zentralen Einflussgröße auf die Landnutzungsentscheidungen von LandwirtInnen geworden (Schmid und Sinabell 2007).

Ausgangspunkt des Projekts war die Überlegung, dass Frauen und Männer in landwirtschaftlichen Betrieben von dem durch Änderungen ökonomischer Rahmenbedingungen (wie Förderungen und Agrarpreise) hervorgerufenen Strukturwandel der Landwirtschaft unterschiedlich betroffen sind (,Gender Gap“). Konkret geht es dabei um erwartbare Disparitäten hinsichtlich Einkommen, Arbeitszeit und Arbeitsbelastung. In einem Simulationsmodell sollten einerseits die durch diese Disparitäten beeinflussten Produktionsentscheidungen erfasst werden, andererseits aber auch deren Auswirkungen auf Landnutzung und ökologische Stoffflüsse wie Stickstoff- und Kohlenstoffflüsse. Ein solches Simulationsmodell, so unsere Erwartung, ist für Wissenschaft und Praxis gleichermaßen interessant, weil es 1) methodisch nachvollziehbare Prognosen über das erwartbare Schicksal landwirtschaftlicher Betriebe in Abhängigkeit von definierten Einflussgrößen und Basisprämissen zulässt und 2) die ökologischen Folgewirkungen der unterschiedlichen Entwicklungsszenarien darstellbar macht.

Bereits bei der Entwicklung der Projektidee waren die Abteilung für ländliche Entwicklung der niederösterreichischen Landesregierung und die Abteilung für Bildung der niederösterreichischen Landwirtschaftskammer maßgeblich beteiligt. So wurde im Rahmen der ersten Gespräche in der Antragsphase die ursprünglich stark auf die Stickstoffproblematik fokussierende Forschungsfrage um Gender-Aspekte erweitert. Die von Seiten der Wissenschaft vorgeschlagene methodische Herangehensweise der partizipativen Modellierung stieß auf reges Interesse. Das heißt, die Initiative für eine transdisziplinäre Erforschung der konkreten Problemstellung war letztlich von einer Allianz aus Wissenschaft, Politik und Interessenvertretung getragen. ${ }^{8}$ 


\subsection{Das Modellkonzept}

Bevor wir nun auf den transdisziplinären Prozess im Konkreten eingehen, wollen wir zunächst den Grundgedanken unseres Modells beschreiben, in dem die Zeitverwendung von Frauen und Männern im landwirtschaftlichen Betrieb im Fokus steht. Zunächst wurden auf Basis ausführlicher Narrationen der Bäuerinnen zeitliche Restriktionen und Limitationen aufgeschlüsselt, die sich aus dem Spannungsverhältnis von arbeits- und lebensweltlichen Anforderungen ergeben. Lebensweltliche Anforderungen ergeben sich in Abhängigkeit von der jeweiligen Lebensphase im Hinblick auf Reproduktions- und Versorgungstätigkeiten im familiären Zusammenhang. Arbeitsweltliche Anforderungen umfassen auch betriebliche und Nebenerwerbstätigkeiten abseits der landwirtschaftlichen Produktion. Beide „Welten“ benötigen Zeit - unabhängig davon, ob sie einkommensrelevant sind oder nicht. Demgegenüber würden beispielsweise Einkommensdaten ausschließlich jene Arbeitsbereiche berücksichtigen, die Lohnarbeit darstellen. Zum einen stellt also die Zeit eine Variable dar, die einen von den konkreten Handlungskontexten abstrahierenden Vergleich zwischen unterschiedlichen Arbeitsbereichen ermöglicht. So ist es möglich, die verschiedenen Tätigkeitsbereiche von Frauen und Männern, alten und jungen Menschen in ihrem Verhältnis und in ihrer Entwicklung darzustellen. Gleichzeitig bietet die Einheit „Stunden“ eine quantitative Größe, die sich in ein formalisiertes Instrument wie ein Modell integrieren lässt und durch die klare Limitierung der zur Verfügung stehenden Zeit auch Grenzen von Handlungsmöglichkeiten aufzeigt.

Ziel des transdisziplinären Prozesses war es nun, Informationen über Entscheidungen bezüglich der landwirtschaftlichen Produktion (wie Fläche oder Viehbesatz vergrößern/ verkleinern, die Fläche intensiver/extensiver bewirtschaften usw.) zu gewinnen. Diese Informationen wurden in Entscheidungsbäume übersetzt. Mit Hilfe von Grenzwerten, die festlegen, ab welcher Arbeitsbelastung (angegeben in Stunden pro Woche) Umstellungen in der landwirtschaftlichen Produktion vorgenommen werden und welcher Art diese Umstellungen - die natürlich ökologische Implikationen durch Intensivierung bzw. Extensivierung der Landnutzung haben - sind, werden „Wenn-Dann“-Funktionen abgeleitet und im Modell simuliert.

Eine wesentliche Herausforderung der Modellierung besteht darin, neben den leicht zugänglichen quantitativen Angaben zum Betrieb, wie etwa Einkommen, landwirtschaftliche Produktion (Flächen, Viehbesatz usw.), auch „qualitative“ Aspekte, insbesondere das Verhalten der AkteurInnen, zu berücksichtigen. Zu diesem Zweck bedarf es einer expliziten Vorstellung darüber, wie Entscheidungen innerhalb landwirtschaftlicher Betriebe getroffen werden, welche, „äußeren“Faktoren, wie z. B. Agrarpreisentwicklungen oder das Förderwesen, aber auch betriebsinterne Entwicklungen wie die Familiensituation oder Zeitpräferenzen das Entscheidungsverhalten einzelner Betriebe beeinflussen. Die oben erwähnten Entscheidungsbäume stellen hierbei die technische Verbindung zwischen quantitativen und qualitativen Daten sowie dem Modell dar. Mithilfe der Ausformulierung von „Wenn-Dann“-Funktionen lässt sich das Entscheidungsverhalten von AkteurInnen im Modell programmieren. Wir haben potenzielle Einflussfaktoren dahingehend analysiert, ob sie relevant für die Entscheidungen in landwirtschaftlichen Betrieben hinsichtlich geschlechtsspezifischer Aspekte sind. Ausgangspunkt dafür war die Differenzierung von Arbeitsbereichen am Hof danach, ob sie zum Großteil von Män- 
nern oder von Frauen übernommen werden. Diese Differenzierung rekurriert auf Studien zur geschlechtsspezifischen Arbeitsteilung in der Landwirtschaft. Während Feldarbeit und Waldarbeit eher von Männern verrichtet wird, sind Frauen meist für die Viehwirtschaft zuständig (Geserick et al. 2008; Oedl-Wieser 1997). Dementsprechend hängt die Entscheidung darüber, ob Arbeiten am Hof weitergeführt, verändert oder aufgegeben werden, von der Zeitintensität dieser Arbeitsbereiche sowie von den individuell und das heißt natürlich auch: je nach Familiensituation - zur Verfügung stehenden Zeitbudgets von Frauen und Männern ab.

In unserem Forschungsprojekt wurden die Betriebe - als Akteure des agentenbasierten Modells - in einem Dreieck angelegt. In diesem Dreieck werden drei Dimensionen von Nachhaltigkeit miteinander in Beziehung gesetzt (Fischer-Kowalski 2002).

Abbildung 1: Das Nachhaltigkeitsdreieck eines landwirtschaftlichen Betriebes

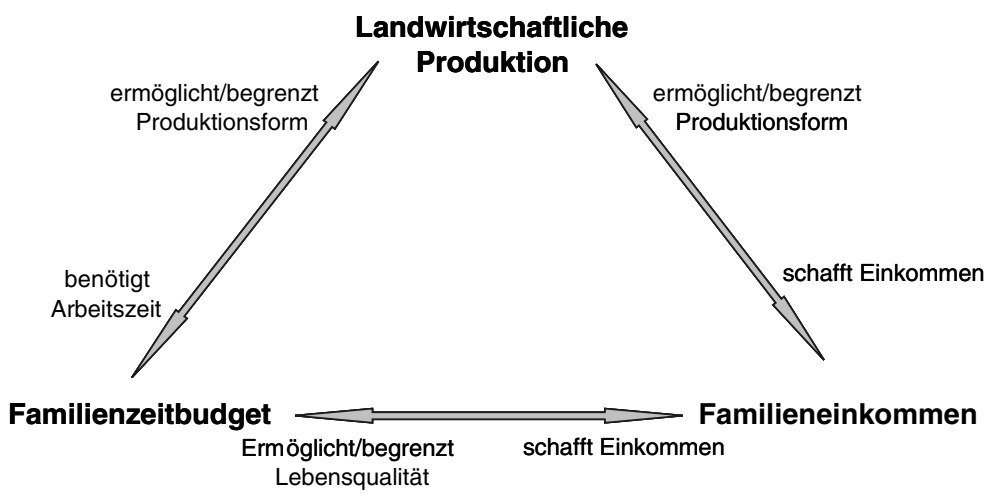

Quelle: eigene Darstellung.

Abbildung 1 zeigt, wie landwirtschaftliche Betriebe gleichsam entlang dieses Dreiecks beschrieben werden können. Die soziale Dimension wird durch die Zeitverwendung der auf einem Hof lebenden Menschen repräsentiert, wobei das Modell die verfügbare Arbeitszeit der erforderlichen Arbeitszeit gegenüberstellt und freie Arbeitskapazität bzw. Überlastung ermittelt. Die ökonomische Dimension wird in Form des Haushaltseinkommens ermittelt, das sich aus der Bewirtschaftung der landwirtschaftlichen Flächen, den Subventionen sowie allen außerlandwirtschaftlichen Tätigkeiten ergibt. Die ökologische Dimension wird durch die Landnutzung und deren Intensität abgebildet. Basierend auf diesen Ergebnissen ist es möglich, Veränderungen in ökologisch relevanten Flüssen, z. B. Stickstoffflüssen, zu berechnen. Jede Veränderung einer dieser Dimensionen, sei sie durch veränderte Rahmenbedingungen wie z. B. Veränderungen des Subventionswesens hervorgerufen oder durch veränderte Präferenzstrukturen des Betriebes, haben jedenfalls auch Auswirkungen auf die anderen beiden Dimensionen. Beispielsweise erzwingt die Notwendigkeit eines höheren Einkommens (etwa auf Grund einer veränderten Familiensituation) bei gleich bleibenden Subventionen und Agrarpreisen einen erhöhten Arbeitszeiteinsatz, der eine intensivere Bewirtschaftung von größe- 
ren Flächen ermöglicht. Dies ist für den einzelnen Betrieb nicht unbegrenzt möglich: Sowohl die verfügbare Arbeitszeit auf einem Hof wie auch die verfügbare Fläche sind limitiert. Darüber hinaus ist das Handeln der Betriebe in der Praxis nicht ausschließlich auf Gewinnmaximierung im klassisch ökonomischen Sinn reduzierbar. Vielmehr handeln die meisten landwirtschaftlichen Betriebe nach Maßgabe einer Mischstrategie der Risiko- und Belastungsvermeidung.

\subsection{Der Prozess der partizipativen Modellentwicklung}

Agentenbasierte Modelle können dazu verwendet werden, Entscheidungen und Handlungen sozialer AkteurInnen zu simulieren; sie ermöglichen auf diese Weise, Veränderungen im Verhalten von AkteurInnen in Abhängigkeit von den Wandlungen etwa der politischen, ökonomischen oder auch natürlichen Rahmenbedingungen (z. B. Klima) abzubilden. Agentenbasierte Modellierung erlaubt es, den Einfluss sozialer Prozesse und individuellen Akteursverhaltens auf die (natürliche) Umwelt zu untersuchen, so z. B. den Einfluss der Landnutzung oder von Düngegewohnheiten von LandwirtInnen auf die Nährstoffdynamik in einer Region (Pahl-Wostl 2002). Andere Studien berichten von der partizipativen Entwicklung agentenbasierter Modelle, mit denen die Auswirkungen von Landmanagement-Entscheidungen auf lokaler Ebene, beispielsweise in Senegal, simuliert wurden (Barreteau et al. 2001; Mendoza et al. 2006). Die computergestützte Modellierung ermöglicht eine quasi-experimentelle Simulation von Prozessen und Szenarien, wobei im Rahmen transdisziplinärer Forschung ganz unterschiedliche, also nicht nur wissenschaftliche Wissensformen Platz finden (Volkery et al. 2008). Abbildung 2 illustriert den Prozess der iterativen Modellentwicklung, wie wir ihn im Projekt zur Anwendung brachten.

Partizipative Modellentwicklung funktioniert im Prinzip nach folgendem Schema: In unterschiedlichen Settings (plenaren Diskussionsrunden, Kleingruppenarbeiten usw.) wird zunächst ein heuristisches Modell über das Zusammenwirken der relevanten AkteurInnen und Rahmenbedingungen in Bezug auf die Fragestellung erstellt. Ein formalisiertes Modell dieser Interaktionsverhältnisse bildet die Grundlage für die Programmierung und Integration von Daten, Entscheidungsbäumen und Schwellenwerten im Simulationsmodell. Das Simulationsmodell wird im Zuge des nächsten Treffens von den AkteurInnen getestet und in Bezug auf die Annahmen und vorläufigen Ergebnisse diskutiert. In diesem rekursiven Prozess wird das ExpertInnenwissen und die Umsetzung dessen im Modell permanent von Seiten der Laien auf Basis des Modellverhaltens geprüft, ergänzt oder bestätigt; die Laienkritik trägt also im Idealfall zu einer Korrektur und Erweiterung verengter ExpertInnenperspektiven bei.

Im Projekt GenderGAP wurde eine transdisziplinäre Arbeitsgruppe mit Bäuerinnen, Expertinnen der niederösterreichischen Landwirtschaftskammer und Wissenschafterinnen aus Soziologie, Biologie und Sozialanthropologie eingerichtet. Die Vertreterinnen der niederösterreichischen Landwirtschaftskammer waren in der Projektanfangsphase für die Zusammenstellung der Arbeitsgruppe verantwortlich. Eingeladen wurden Landwirtinnen zwischen 25 und 60 Jahren, die unterschiedliche Produktionsbereiche repräsentierten. Das vorhandene Wissen um ein prinzipielles Interesse der Bäuerinnen als 


\section{Abbildung 2: Prozess der partizipativen Modellierung}

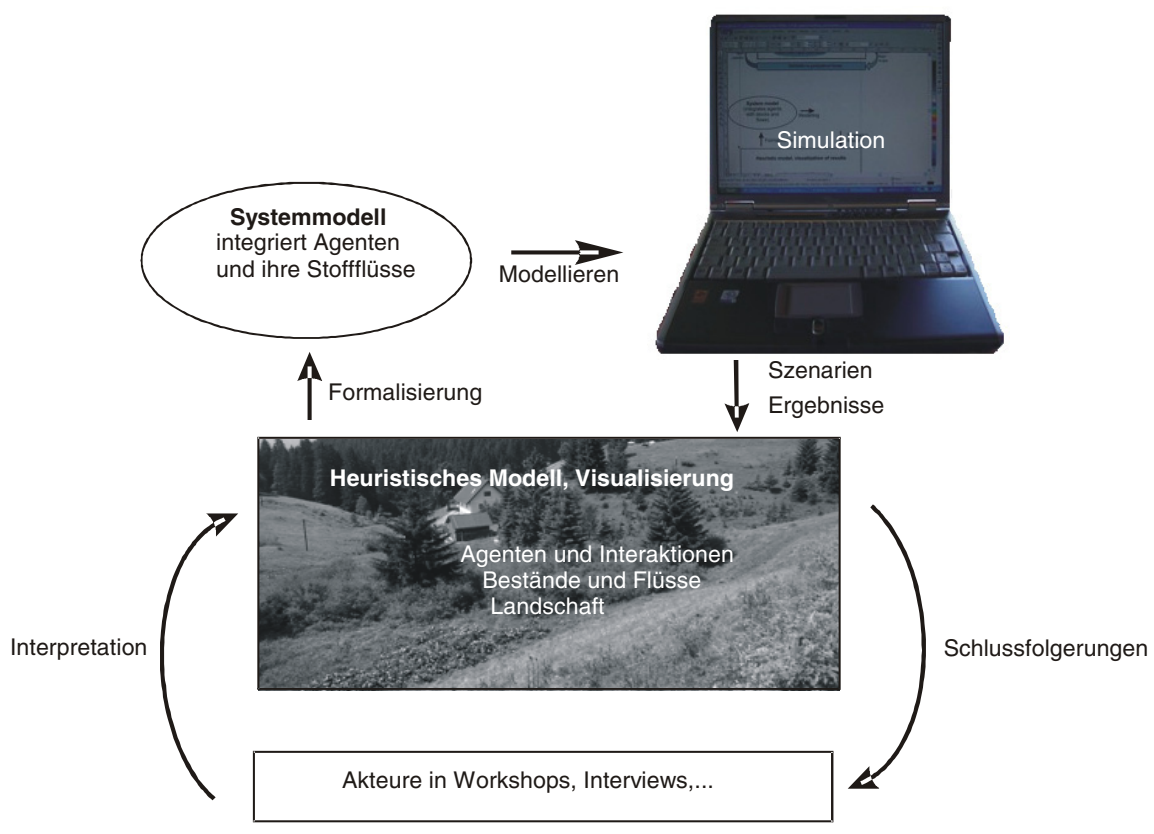

Quelle: Eigene Darstellung nach Berger 2004 und Singh et al. 2010

Mitglieder der ARGE Bäuerinnen gewährleistete, dass es sich um durchaus motivierte und für derartige Projekte offene Teilnehmerinnen handelte. Im Laufe von 18 Monaten kam diese Gruppe fünf Mal zu halbtägigen Workshops in der niederösterreichischen Landwirtschaftskammer zusammen. In den ersten beiden Workshops wurden die Grundannahmen des Modells bezüglich maximaler Arbeitsbelastungen in unterschiedlichen Arbeitsbereichen und Mindesteinkommen erarbeitet. Dies geschah einerseits in Diskussionsrunden, in welchen gemeinsam über die Zusammenhänge von Arbeitszeit, Einkommen und Betriebsumstellungen debattiert wurde. Andererseits wurden auch Kleingruppenarbeiten abgehalten, deren Aufgabe es war, quantitative Angaben darüber zu machen, ab welcher Arbeitsbelastung (in Stunden pro Woche) Veränderungen im Arbeits- und Produktionsprozess notwendig sind und welche Auswirkungen diese Neuerungen auf die Arbeitsbelastung haben. Im dritten und vierten Workshop wurden einerseits erste Versionen des Computermodells vorgestellt, von den Akteurinnen in Kleingruppen auf Computern ausprobiert und anschließend diskutiert. Andererseits war es Ziel der beiden Workshops, deskriptive Szenarien zu entwickeln, die das Modell berechnen können soll. Die Szenarienergebnisse sowie das beinahe fertige Modell wurden den Teilnehmerinnen in einem Abschlussworkshop präsentiert und von diesen getestet. Kleingruppen von zwei bis drei Teilnehmerinnen, die jeweils einen Computer zur Verfügung hatten, beobachteten anhand der auf der Bildschirmoberfläche befindlichen Graphen (siehe Abbildung 3), wie sich unter unterschiedlichen ökonomischen Rahmenbe- 
dingungen Indikatoren wie beispielsweise jährliches Familieneinkommen (in Euro), jährliche Arbeitsbelastung pro Betrieb und Person (in Stunden) und Entwicklung der Flächenkategorien Acker, Wald und Grünland (in Hektar) und Intensität der Landnutzung (kg Stickstoff pro Hektar) der Region über einen Zeitraum von 20 Jahren entwickelten. Sofern eine plausible Erklärung der Ergebnisse nicht möglich schien, wurden nochmals die Modellannahmen in Frage gestellt und gewisse Parameter oder Schwellenwerte in den Entscheidungsbäumen modifiziert.

Aufgabe der involvierten Sozialwissenschafterinnen war es, die durch Gruppendiskussionen angeregten Narrationen der Bäuerinnen zum Zweck der Modellbildung auszuwerten und aufzubereiten. Außerdem oblagen ihnen die Organisation der Reflexionsprozesse in den Arbeitsgruppen sowie die Gestaltung des transdisziplinären Prozesses.

Die Frage nach dem entsprechenden Handeln und den Präferenzen der Personen in den Betrieben war wesentlicher Gegenstand des Prozesses der partizipativen Entwicklung und Verfeinerung dieses Modells. Den Beteiligten war vor allem am Anfang des Prozesses die Sinnhaftigkeit der Entwicklung eines solchen Modells nicht zugänglich. Gerade Fragen von Seiten der Forscherinnen, die auf die Quantifizierung bestimmter Größen bei Betriebsumstellungen abzielten (z. B. Arbeitsbelastung in Stunden, Wahrscheinlichkeiten einzelner Maßnahmen), erregten mitunter Unwillen. Allerdings trug das im Zug der kommunikativen Prozesse stabilisierte Vertrauen dazu bei, dass sich die Beteiligten in zunehmendem Maße auf diese „Übungen“ einließen. Am Ende des Prozesses überwog eine positive Wahrnehmung, weil eine derartig „objektivierte“ Auseinandersetzung mit dem eigenen Alltag auf eine quasi experimentelle Weise auch neue Einsichten für die Beteiligten erbrachte, und zwar über die den Entscheidungsprozessen inhärenten Kriterien und Logiken. Auf Basis dieser Rationalitätsgewinne wurde explizit die Erwartung geäußert, mit zukünftigen Änderungen von Rahmenbedingungen wesentlich bewusster umgehen zu können (vgl. auch Fraser et al. 2006).

\subsection{Szenarien und Ergebnisse}

Wissenschaftliches Ergebnis der partizipativen Modellentwicklung ist ein Computermodell, das ohne größere Anleitung auch von Laien genutzt werden kann. Man kann beispielsweise die dem Modell zugrunde liegenden Annahmen ändern und dann beobachten, welche Auswirkungen diese veränderten Annahmen auf die Entwicklung der landwirtschaftlichen Betriebe haben (Abbildung 3).

Die Gruppe entwickelte zwei Extremszenarien: Das „Nachhaltigkeitsszenario“ beschreibt einen deutlichen Anstieg von Subventionen in erster Linie nachhaltiger Produktionsweisen. Zugleich erlaubt die Entwicklung des Agrarpreises vor allem für Bioprodukte eine gewisse Unabhängigkeit der landwirtschaftlichen Betriebe von Förderungen. Diese Entwicklungen garantieren den Betrieben bei gleich bleibender Größe (Fläche und Viehbesatz) und nachhaltiger Produktionsweise (wenig Düngerbeigaben) mehr Einkommen pro Einheit (Hektar oder Vieheinheit). Neben der positiven Entwicklung in der Landwirtschaft bietet die Region mehr Infrastruktureinrichtungen für Pflege und Betreuung von Kindern und älteren Menschen. Außerdem stehen genügend und vor allem attraktive Arbeitsplätze in der näheren Umgebung zur Verfügung, die Frauen wie Män- 


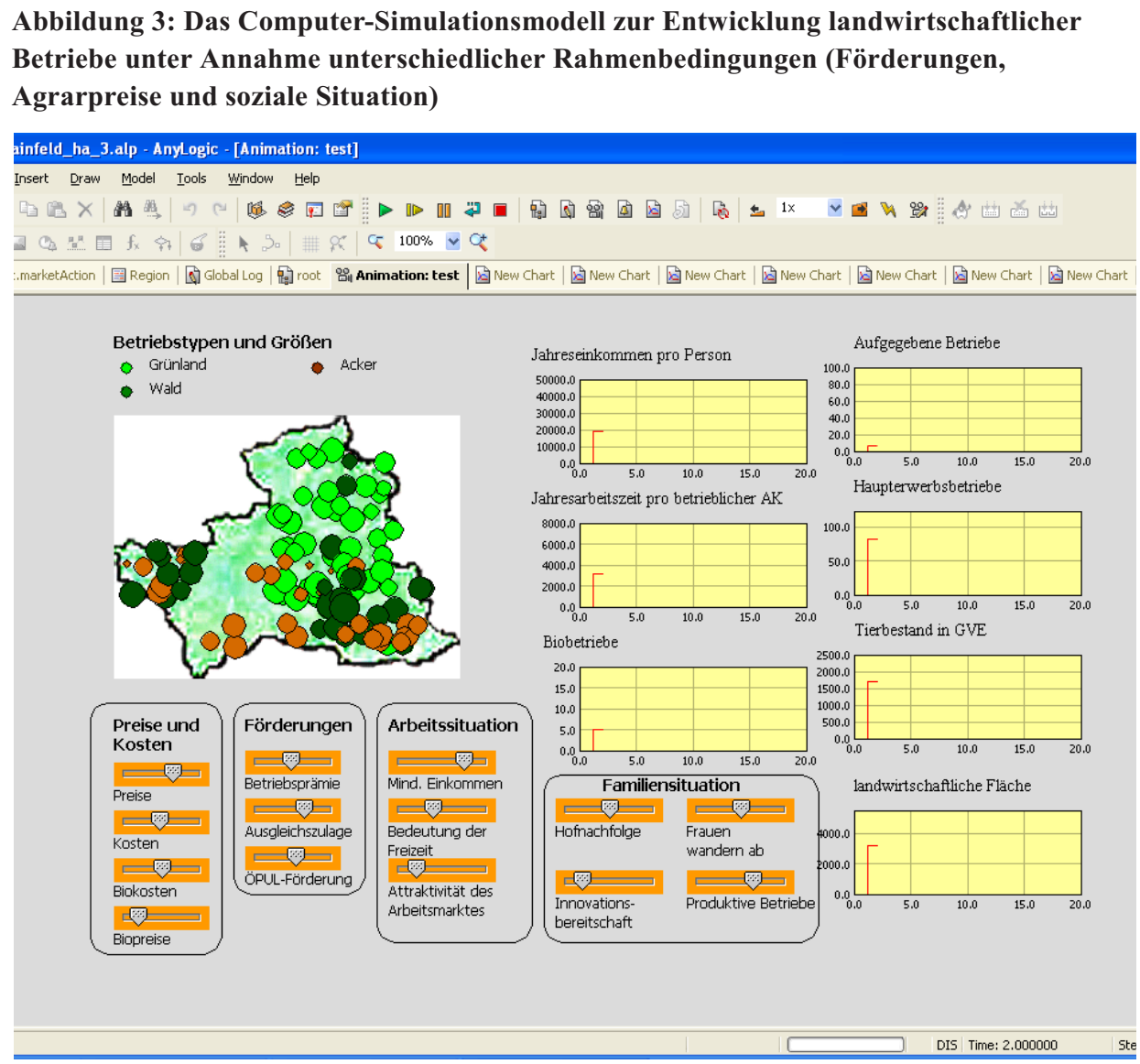

Quelle: eigene Darstellung

nern von landwirtschaftlichen Betrieben eine attraktive außerlandwirtschaftliche Einkommensmöglichkeit bieten.

Eine Alternative stellt das „Globalisierungsszenario“ dar. In diesem Gefahrenszenario wird eine Zukunft entworfen, in der sowohl die Subventionen wie auch die Agrarpreise massiv fallen und landwirtschaftliche Produktion bei gleich bleibender Größe ein kaum ausreichendes Familieneinkommen gewährleistet. Zusätzlich stagniert die Regionalentwicklung in den ländlichen Regionen, es stehen weder ausreichend Arbeitsplätze noch zufrieden stellende Infrastruktureinrichtungen zur Verfügung. Da die Gruppe bereits viel über die Annahmen und Limitierungen des Modells wusste, war die Diskussion über die narrativen Szenarien deutlich auf die Möglichkeiten des Modells fokussiert. Dies markiert eine klare Grenze und einen potenziellen Blindfleck einer solchen partizipativen Modellentwicklung: Die Gruppe ist von Beginn an gehalten, sich konstruktiv auf bestimmte Parameter und Wechselwirkungen zu einigen und die Diskussion darauf zu beschränken. 
Die partizipativ entwickelten Szenarien wurden im Modell durch Veränderungen von Faktoren simuliert, die sich auf eine der drei Dimensionen im Nachhaltigkeitsdreieck des landwirtschaftlichen Betriebs direkt auswirken (in erster Linie die ökonomische). Die landwirtschaftliche Nutzfläche geht in allen Szenarien zurück. Der Rückgang der Anzahl der landwirtschaftlichen Betriebe ist dramatisch.

Gemäß dem vorliegenden Modell zieht eine Stärkung des regionalen Arbeitsmarktes - wie es im „Nachhaltigkeitsszenario“ angenommen wird - nicht etwa Arbeitskraft aus der Landwirtschaft ab, sondern trägt vielmehr dazu bei, die Landwirtschaft aufrecht zu erhalten; schließlich wird es möglich, den Betrieb durch einigermaßen attraktive außerlandwirtschaftliche Arbeit zu stabilisieren. Um allerdings einer stärkeren Belastung der Bäuerinnen entgegenzuwirken, ist es gleichzeitig notwendig, die regionale Infrastruktur entsprechend attraktiv zu gestalten, und das heißt: für ausreichende Betreuungsmöglichkeiten für Kinder und alte Menschen zu sorgen sowie bei Versorgungsleistungen sowie bei der Entwicklung von neuen landwirtschaftlichen Arbeitszeitmodellen und innovativer Arbeitsorganisation (z. B. in Form von Kooperationen) zu unterstützen. All dies bezeichnet zum einen die für uns maßgeblichen Punkte, an denen eine nachhaltigkeitsorientierte Politik anzusetzen hätte - und unterstreicht gleichzeitig die Bedeutung von Gender-Aspekten im Rahmen einer solchen Politik (Smetschka et al. 2008).

\section{Resümee}

Auch und gerade im Nachhaltigkeitsbereich sollte die Diskussion darüber intensiviert werden, worin der Mehrwert einer ,von oben“ initiierten Partizipation in einem Zeitalter nachlassender Partizipationsansprüche (klassisch dazu: Putnam 1995) bestehen könnte. Schließlich ist Transdisziplinarität - im Sinne der Beteiligung außerwissenschaftlicher AkteurInnen - in diesem Bereich geradezu zum Forschungsideal avanciert. Transdisziplinäre Forschung unterliegt spezifischen Gelingensbedingungen, nicht zuletzt weil Partizipation in diesem Fall meist von Seiten der Wissenschaft und/oder Politik initiiert und organisiert wird. Diese experimentelle Partizipation unterscheidet sich in ihrer Funktion und Leistungsfähigkeit von anderen, etwa protestförmigen Beteiligungsformen, wie sie für die Hochphase der Ökologiebewegung typisch waren. Eine weitreichende Politisierungswirkung wird man von experimenteller Partizipation nicht erwarten dürfen. Beteiligungsexperimente können jedoch, wie unsere Studie gezeigt hat, Rationalitätsgewinne realisieren. Allein die Unterstellung eines derartigen Mehrwerts von Partizipation ist keineswegs selbstverständlich. Anhand eines sozialökologischen Forschungsprojekts, dessen methodischer Kern eine partizipative Modellentwicklung darstellte, haben wir gezeigt, dass die Beteiligung außerwissenschaftlicher AkteurInnen im Forschungsprozess dann die erhofften Rationalitätsgewinne realisiert, wenn Eigenmotivation, eine gewisse Betroffenheit und ein bestimmtes Spezialwissen (etwa Erfahrungswissen) vorausgesetzt werden kann. Im konkreten Fall wurde dieses Erfahrungswissen über Produktionsentscheidungen im landwirtschaftlichen Betrieb in einem rekursiven Prozess in ein Modell übersetzt. Nicht zuletzt trug das Wissen der ExpertInnen und Betroffenen maßgeblich zur Ausformulierung von Szenarien und Handlungsmöglichkeiten bei. Breitere Beteiligung ist überdies für das Testen und Verfeinern von formalen Model- 
len von Nutzen. Dass all diese Leistungen in der Sachdimension auch entsprechende Leistungen in der Sozialdimension voraussetzen, haben wir erwähnt.

Diese positive Einschätzung sollte freilich nicht darüber hinwegtäuschen, dass mit dem experimentellen Design natürlich auch Restriktionen verbunden sind. So übt das gemeinsame und übergreifende Ziel einer modellhaften Konstruktion von Wirklichkeit auf alle Beteiligten den Zwang aus, sich konstruktiv auf die Struktur des Modells und seine zentralen Elemente zu beziehen. Zwar ist Kritik natürlich möglich (und erwünscht), doch muss diese Kritik, um handlungsrelevant werden zu können, im Fortgang des Prozesses sich positiv auf jene Basisprämissen beziehen, die für die Entwicklung einer Grobstruktur des Modells maßgeblich waren. Grundsätzliche Perspektivenwechsel oder thematische Korrekturen erlaubt das Modell nur noch in einem geringen Ausmaß. Im konkreten Beispiel etwa hätte ein Abrücken von den am Anfang des Aushandlungsprozesses definierten Entscheidungsparametern (wie Zeitverwendung, Bildung und Hofnachfolge) den Erfolg des Projektes gravierend gefährdet, da kein anwendungsfähiges Modell hätte finalisiert werden können. Diese - im Partizipationsprozess sich durchsetzende - Orientierung auf einen interaktiv darstellungsfähigen und technisch anwendbaren „Output“ markiert Grenzen der Partizipation im Modellierungsbereich.

\section{Anmerkungen}

1 Hinter dem unscheinbaren Begriff der Umweltfragen verbirgt sich in der Regel eine komplexe Problemkonstellation. Denn die Umwelt wird ja oft im Kontext konkreter Technisierungsprojekte oder bestimmter Modernisierungsmodelle zum Thema.

2 Zur Unterscheidung zwischen ,invited“ und „uninvited participation“ vgl. Wynne (2007).

3 Antworten auf diese Frage hat Luhmann in verschiedenen Anläufen durchgespielt, wobei sich seine Argumentation im Laufe der Zeit nicht wesentlich wandelt. Vgl. Luhmann (1986, S. 227 f.; 1991, S. 135 ff.; 1996; 1997, S. 847 ff.). Anregend dazu auch Virgl (2011).

4 Beispiel „Brent Spar“ (1995): Dieser im Atlantik schwimmende Öltank des Shell-Konzerns wurde aufgrund einer Medienkampagne von Greenpeace, die auf falschen Messdaten beruhte, an Land entsorgt. Die Versenkung im Meer wäre die ,umweltfreundlichere“ Variante gewesen - ganz so, wie Shell auf Basis eigener Zahlen argumentiert hatte.

5 Zu Format und Geschichte dieser in Dänemark entwickelten und erstmals 1987 eingesetzten Beteiligungsmethode vgl. Joss (2003).

6 Andere, z. B. differenzierungstheoretisch argumentierende Positionen, die partizipative Forschung als „Transwissenschaft“ beschreiben (Bora 2010), spielen in diesen Umweltdiskursen keine Rolle.

7 Dieses Projekt mit dem Kürzel „GenderGAP“ wurde zwischen 2005 und 2007 durchgeführt; die Projektleitung lag bei Barbara Smetschka.

8 Interessenvertretung und Bildungsbereich behielten eine zentrale Rolle bis über das Projektende hinaus. So wurde im Anschluss - und ohne weitere Beteiligung der Wissenschafterinnen - eine Arbeitsgruppe zu „Frauen in der Landwirtschaft“ in der niederösterreichischen Landeslandwirtschaftkammer eingerichtet. 


\section{Literatur}

Abels, Gabriele und Alfons Bora. 2004. Demokratische Technikbewertung. Bielefeld: transcript. Barreteau, Olivier, Francois Bousquet und Jean-Marie Attonaty. 2001. Role-playing games for opening the black box of multi-agent systems: method and lessons of its application to Senegal River Valley irrigated systems. Journal of Artificial Societies and Social Simulation 4. http:// ideas.repec.org/a/jas/jasssj/2000-18-1.html.

Bechtold, Ulrike, Michael Ornetzeder und Mahshid Sotoudeh. 2011. WWViews in Austria - reflections on a citizen consultation project from a Technology Assessment perspective. In: Global Deliberation: A World of Opportunity, hrsg. Mikko Rask, Richard Worthington und Minna Lammi. London: Earthscan (forthcoming).

Beck, Ulrich. 1986. Risikogesellschaft. Auf dem Weg in eine andere Moderne. Frankfurt/M.: Suhrkamp.

Berger, Thomas. 2004. Agentenbasierte Modellierung von Landnutzungsdynamiken und Politikoptionen; Agent-based Modeling of Land Use Dynamics and Policy Options. Agrarwirtschaft 53(2): 77-87.

BMLFUW, Bundesministerium für Land- und Forstwirtschaft, Umwelt und Wasserwirtschaft. 2010. Grüner Bericht 2010. Wien: BMLFUW.

Bogner, Alexander. 2005. Grenzpolitik der Experten. Vom Umgang mit Ungewissheit und Nichtwissen in pränataler Diagnostik und Beratung. Weilerswist: Velbrück Wissenschaft.

Bogner, Alexander. 2010. Partizipation als Laborexperiment. Paradoxien der Laiendeliberation in Technikfragen. Zeitschrift für Soziologie 39: 87-105.

Bogner, Alexander, Karen Kastenhofer und Helge Torgersen. 2010. Inter- und Transdisziplinarität im Wandel? Neue Perspektiven auf problemorientierte Forschung und Politikberatung. Baden-Baden: Nomos.

Bonß, Wolfgang, Rainer Hohlfeld und Regine Kollek. 1993. Wissenschaft als Kontext-Kontexte der Wissenschaft. Hamburg: Junius.

Bora, Alfons. 2010. Wissenschaftliche Politikberatung und die disziplinären Grundlagen der Wissenschaft. In: Inter- und Transdisziplinarität im Wandel? Neue Perspektiven auf problemorientierte Forschung und Politikberatung, hrsg. Alexander Bogner, Karen Kastenhofer und Helge Torgersen, 25-55. Baden-Baden: Nomos.

Brand, Karl-Werner. 2008. Umweltbewegung. In: Die Sozialen Bewegungen in Deutschland seit 1945. Ein Handbuch, hrsg. Roland Roth und Dieter Rucht, 219-244. Frankfurt/New York: Campus.

Collins, Harry und Robert Evans. 2007. Rethinking Expertise. Chicago and London: University of Chicago Press.

Dessai, Suraje und Mike Hulme. 2004. Does climate adaptation policy need probabilities? Climate Policy 4: 107-128.

Dröge, Kai und Chantal Magnin. 2010. Integration durch Partizipation? Zum Verhältnis von formeller und informeller Bürgerbeteiligung am Beispiel der Stadtplanung. Zeitschrift für Rechtssoziologie 1: 103-121.

Epstein, Steven. 1996. The Construction of Lay Expertise: AIDS Activism and the Forging of Credibility in the Reform of Clinical Trials. Science, Technology \& Human Values 20: 408-437.

Few, Roger, Katrina Brown und Emma L. Tompkins. 2007. Public participation and climate change adaptation: avoiding the illusion of inclusion. Climate Policy 7: 46-59.

Fischer-Kowalski, Marina. 2002. Das magische Dreieck von Nachhaltigkeit: Lebensqualität, Wohlstand und ökologische Verträglichkeit. In: Stadt und Nachhaltigkeit, hrsg. Arnold Klotz, 25-41. Wien/New York: Springer.

Fischer-Kowalski, Marina und and Helmut Haberl. 2007. Socioecological transitions and global change: Trajectories of Social Metabolism and Land Use. Cheltenham, UK and Northhampton, USA: Edward Elgar. 
Fraser, Evan D. G., Andrew J. Dougill, Warren E. Mabee, Mark Reed und Patrick McAlpine. 2006. Bottom up and top down: Analysis of participatory processes for sustainability indicator identification as a pathway to community empowerment and sustainable environmental management. Journal of Environmental Management 78: 114-127.

Gaube, Veronika, Christina Kaiser, Martin Wildenberg, Heidi Adensam, Peter Fleissner, Johannes Kobler, Juliana Lutz, Andreas Schaumberger, Jakob Schaumberger, Barbara Smetschka, Angelika Wolf, Andreas Richter und Helmut Haberl. 2009. Combining agent-based and stockflow modelling approaches in a participative analysis of the integrated land system in Reichraming, Austria. Landscape Ecology 24: 1149-1165.

Gavelin, Karin, Richard Wilson und Robert Doubleday. 2007. Democratic Technologies? The final report of the Nanotechnology Engagement Group (NEG). London: Involve.

Geserick, Christine, Olaf Kapella und Markus Kaindl. 2008. Situation der Bäuerinnen in Österreich 2006. Ergebnisse der repräsentativen Erhebung. Informationsdienst des Österreichischen Instituts für Familienforschung 68. Wien: Österreichisches Institut für Familienforschung.

Gibbons, Michael, Camille Limoges, Helga Nowotny, Simon Schwartzman, Peter Scott und Martin Trow (1994). The new production of knowledge: the dynamics of science and research in contemporary societies. London: Sage.

Groß, Matthias, Holger Hoffmann-Riem und Wolfgang Krohn. 2003. Realexperimente: Robustheit und Dynamik ökologischer Gestaltungen in der Wissensgesellschaft. Soziale Welt 54: 241-258.

Groß, Matthias, Holger Hoffmann-Riem und Wolfgang Krohn. 2005. Realexperimente. Ökologische Gestaltungsprozesse in der Wissensgesellschaft. Bielefeld: transcript.

Haberl, Helmut, Karl-Heinz Erb, Fridolin Krausmann, Heidi Adensam und Niels B. Schulz. 2003. Land-Use Change and Socioeconomic Metabolism in Austria. Part II: Land-Use Scenarios for 2020. Land Use Policy 20: 21-39.

Hirsch-Hadorn, Gertrude, David Bradley, Christian Pohl, Stephan Rist und Urs Wiesmann. 2006. Implications of transdisciplinarity for sustainability research. Ecological Economics 60: 119-128.

Jantsch, Erich. 1972. Towards Interdisciplinarity and Transdisciplinarity in Education and Innovation. In: Innovation, hrsg. Centre for Educational Research and Interdisciplinarity. Problems of Teaching and Research in Universities. Paris: Organization for Economic Cooperation and Development.

Jasanoff, Sheila. 2003. Breaking the Waves in Science Studies: Comment on H. M. Collins and Robert Evans, ,The Third Wave of Science Studies'. Social Studies of Science 33: 389-400.

Jørgensen, Ulrik und Peter Karnøe. 1995. The Danish Wind-Turbine Story: Technical Solutions to Political Visions? In: Managing Technology in Society: The Approach of Constructive Technology Assessment, hrsg. Arie Rip, Thomas Misa und Johan Schot, 57-82. London: Pinter.

Joss, Simon. 2003. Zwischen Politikberatung und Öffentlichkeitsdiskurs - Erfahrungen mit Bürgerkonferenzen in Europa. In: Bürgerkonferenz: Streitfall Gendiagnostik. Ein Modellprojekt der Bürgerbeteiligung am bioethischen Diskurs, hrsg. Silke Schicktanz und Jörg Naumann, 15-35. Opladen: Leske + Budrich.

Krausmann, Fridolin, Helmut Haberl, Niels B. Schulz, Karl-Heinz Erb, Ekkehard Darge und Veronika Gaube. 2003. Land-use change and socio-economic metabolism in Austria. Part I: driving forces of landuse change: 1950-1995. Land Use Policy 20: 1-20.

Krohn, Wolfgang. 2007. Realexperimente - Die Modernisierung der ,offenen Gesellschaft` durch experimentelle Forschung. Erwägen Wissen Ethik 18: 343-356.

Krohn, Wolfgang und Johannes Weyer. 1989. Gesellschaft als Labor. Die Erzeugung sozialer Risiken durch experimentelle Forschung. Soziale Welt 40: 349-373. 
Kurath, Monika und Priska Gisler. 2009. Informing, involving or engaging? Science communication, in the ages of atom-, bio- and nanotechnology. Public Understanding of Science 18: 559-573.

Luhmann, Niklas. 1986. Ökologische Kommunikation - Kann die moderne Gesellschaft sich auf ökologische Gefährdungen einstellen? Opladen: Westdeutscher Verlag.

Luhmann, Niklas. 1991. Soziologie des Risikos. Berlin/New York: de Gruyter.

Luhmann, Niklas. 1996. Protest. Systemtheorie und soziale Bewegungen, hrsg. Kai-Uwe Hellmann. Frankfurt/M.: Suhrkamp.

Luhmann, Niklas. 1997. Die Gesellschaft der Gesellschaft. Frankfurt/M.: Suhrkamp.

Mendoza, Guillermo A. und Ravi Prabhu. 2006. Participatory modeling and analysis for sustainable forest management: Overview of soft system dynamics models and applications. Forest Policy and Economics 9: 179-196.

Menz, Wolfgang. 2009. Die Legitimität des Marktregimes. Leistungs- und Gerechtigkeitsorientierungen in neuen Formen betrieblicher Leistungspolitik. Wiesbaden: VS.

Newig, Jens, Helmut Haberl, Claudia Pahl-Wostl und Dale Rothman. 2008. Formalised and NonFormalised Methods in Resource Management, Knowledge and Learning in Participatory Processes. An Introduction. Systemic Practice and Action Research 21: 381-387.

Nowotny, Helga, Peter Scott und Michael Gibbons. 2001. Re-Thinking Science - Knowledge and the Public in an Age of Uncertainty. Cambridge: Polity Press.

Oedl-Wieser, Theresia. 1997. Emanzipation der Frauen am Land. Eine explorative Studie über Ambivalenzen und Lebenszusammenhänge. Forschungsbericht Nr. 40 der Bundesanstalt für Bergbauernfragen. Wien.

Ornetzeder, Michael und Harald Rohracher. 2006. User-led Innovations and Participation Processes: Lessons from Sustainable Energy Technologies. Energy Policy 34: 138-150.

Pahl-Wostl, Claudia. 2002. Agent Based Simulation in Integrated Assessment and Resources Management. In: Integrated Assessment and Decision Support-proceedings of the $1^{\text {st }}$ Biennial Meeting of the International Environmental Modelling and Software Society, Vol. 2. SEA, Como., hrsg. Aandrea Rizzoli und Anthony Jakeman, 239-244.

Pohl, Christian, Gabriela Wülser und Gertrude Hirsch-Hadorn. 2010. Transdisziplinäre Nachhaltigkeitsforschung: Kompromittiert die Orientierung an der gesellschaftlichen Leitidee den Anspruch als Forschungsform? In: Inter- und Transdisziplinarität im Wandel? Neue Perspektiven auf problemorientierte Forschung und Politikberatung, hrsg. Alexander Bogner, Karen Kastenhofer und Helge Torgersen, 123-143. Baden-Baden: Nomos.

Putnam, Robert. 1995. Bowling Alone: America's Declining Social Capital. Journal of Democracy $6: 65-78$

Reed, Mark S., Evan D. G. Fraser und Andrew J. Dougill. 2006. An adaptive learning process for developing and applying sustainability indicators with local communities. Ecological Economics 59: 406-418.

Rohracher, Harald. 2002. A Socio-Technical Mapping of Domestic Biomass Heating Systems in Austria. Bulletin of Science, Technology and Society 22: 474-483.

Roose, Jochen. 2010. Der endlose Streit um die Atomenergie. Konfliktsoziologische Untersuchung einer dauerhaften Auseinandersetzung. In: Technik- und Umweltkonflikte, hrsg. Peter Feindt und Thomas Saretzki, 79-103. Wiesbaden: VS.

Rucht, Dieter. 2003. The Changing Role of Political Protest Movements. In: Germany Beyond the Stable State, Special Issue West European Politics, Vol. 26, No. 4, hrsg. Herbert Kitschelt, and Wolfgang Streek, 153-176.

Rucht, Dieter. 2008. Anti-Atomkraftbewegung. In: Die sozialen Bewegungen in Deutschland seit 1945. Ein Handbuch, hrsg. Roland Roth und Dieter Rucht, 245-266. Frankfurt/New York: Campus. 
Schmid, Martin und Ortrun Veichtlbauer. 2007. Vom Naturschutz zur Ökologiebewegung. Umweltgeschichte Österreichs in der Zweiten Republik. Innsbruck: Studienverlag.

Schmid, Erwin und Franz Sinabell. 2007. On the choice of farm management practices after the reform of the Common Agricultural Policy 2003. Journal of Environmental Management 82: $332-340$.

Schot, Johan und Arie Rip. 1996. The Past and Future of Constructive Technology Assessment. Technological Forecasting and Social Change 54: 251-268.

Smetschka, Barbara, Veronika Gaube und Juliana Lutz. 2008. Gender als forschungsleitendes Prinzip in der transdisziplinären Nachhaltigkeitsforschung. In: Transdisziplinäre Praxis, hrsg. Elisabeth Reitinger, 23-34. Heidelberg: Carl-Auer Verlag.

Take, Ingo. 2002. NGOs im Wandel. Von der Graswurzel auf das diplomatische Parkett. Wiesbaden: Westdeutscher Verlag.

UNFCCC. 1992. United Nations Framework Convention on Climate Change. http://unfecc.int/resource/docs/convkp/conveng.pdf.

Virgl, Christoph J. 2011. Protest in der Weltgesellschaft. Wiesbaden: VS.

Volkery, Axel, Teresa Ribeiro, Thomas Henrichs und Ybele Hoogeveen. 2008. Your Vision or My Model? Lessons from Participatory Land Use Scenario Development on an European Scale. Systemic Practice and Action Research 21: 459-477.

Walk, Heike. 2010. What's Holding up the Climate Movement? A Look at Germany. The Journal of Transdisciplinary Environmental Studies 9. http://www.journal-tes.dk/.

Wilsdon, James und Rebecca Willis. 2004. See-through Science: Why public engagement needs to move upstream. London: Demos.

Wynne, Brian. 1996. Misunderstood misunderstandings - social identities and public uptake of science. In: Misunderstanding science? The public reconstruction of science and technology, hrsg. Alan Irwin, and Brian Wynne, 19-46. Cambridge: University Press.

Wynne, Brian. 2007. Public Participation in Science and Technology: Performing and Obscuring a Political-Conceptual Category Mistake. East Asian Science, Technology and Society: an International Journal 1: 99-110.

Alexander Bogner, PD Dr., Soziologe, ist Senior Researcher an der Österreichischen Akademie der Wissenschaften, Assistenzprofessor am Institut für Soziale Ökologie an der Alpen-Adria Universität und Lektor am Institut für Soziologie der Universität Wien. Arbeitsschwerpunkte: Wissenschafts- und Technikforschung, Umweltsoziologie, Methoden empirischer Sozialforschung. Veröffentlichungen: Let's disagree! Talking Ethics in Technology Controversies, in: Science, Technology \& Innovation Studies (2010); Partizipation als Laborexperiment. Paradoxien der Laiendeliberation in Technikfragen, in: Zeitschrift für Soziologie (2010); Ethisierung und die Marginalisierung der Ethik, in: Soziale Welt (2009).

Veronika Gaube, Mag. Dr., Ökologin, ist Senior Researcher und Lehrbeauftragte am Institut für Soziale Ökologie an der Alpen-Adria Universität. Arbeitsschwerpunkte: Integrierte sozial-ökologische Modellierung, partizipative Modellentwicklung, Landnutzung (lokal bis global). Artikelrelevante Veröffentlichungen: Combining agent-based and stock-flow modelling approaches in a participative analysis of the integrated land system in Reichraming, Austria, in: Landscape Ecology (2009); The Role of Formalisation, Participation and Context in the Success of Public Involvement Mechanisms in Resource Management, in: Systemic Practice and Action Research (Newig et al., 2008). 
Barbara Smetschka, Mag., Sozialanthropologin, ist Senior Researcher und Lehrbeauftragte am Institut für Soziale Ökologie an der Alpen-Adria Universität. Arbeitsschwerpunkte: inter- und transdisziplinäre Forschung, Gender und Nachhaltigkeit, partizipative Modellentwicklung, Zeitverwendung aus sozial-ökologischer Perspektive. Artikelrelevante Veröffentlichungen: Integration der Genderperspektive im Nachhaltigkeitsdreieck mittels Zeitverwendung. In: Jahrbuch der Österreichischen Gesellschaft für Agrarökonomie (2009). Gender als forschungsleitendes Prinzip in der transdisziplinären Nachhaltigkeitsforschung. In: Transdisziplinäre Praxis (Hg. Reitinger, Elisabeth, Carl-Auer Verlag, 2008). 